\title{
A Comparative Analysis of CBO over MVG to Improve Quality of Software
}

\author{
Dharmendra Lal Gupta \\ Deptt. of Computer Science \& Engg. \\ KNIT Sultanpur, UP, India \\ Member IEEE
}

\author{
Vikash Chauhan \\ Deptt. of Computer Science \& Engg. \\ KNIT Sultanpur, UP, India
}

\begin{abstract}
This paper describes the relationship between two metrics CBO (Coupling between Objects) and MVG (McCabe's Cyclomatic Complexity). CBO is the object oriented CK metric and MVG is the complexity metric. Here authors have analysed three projects with different complexity level. Authors also have calculated CBO and MVG metrics of these projects and found the correlation between these two metrics. We have found that on increasing CBO, MVG also increases in polynomial form. This correlation study provides an optimal value of $\mathrm{CBO}$ up to that value, software will be a quality software.
\end{abstract}

\section{Keywords}

$\mathrm{CBO}$, MVG, Correlation between $\mathrm{CBO}$ and MVG.

\section{INTRODUCTION}

Delivering high quality and highly reliable software is a mandatory goal for software development companies. Basically quality of software depends on its source code. If source code of any software is written in good way then its quality will increase. Thus in this way the amount of resources needed to support the software will reduce. So today's focus is on enhancing the software development process [1].

There is an critical need for presenting the of various statistics for evaluating the change-proneness for knowing what is wrong with ones projects in progress so that there is no burn out between the stakeholder working in the life cycle of the application. Since, components of software are like organic compounds that change internally and externally with multiple environmental and business reasons. The major concern is maintenance and further development of the software without conflicts, issues and bugs. Changeability and upgradeability in software is very risky so to reduce this risk and to measure of impact of changes one have to maintain ones source code.

Changeability is the ease with which a source code can be changed. It is evaluated through metrics calculated from the history of changes made. These metrics reflect how well or bad is the change for the project in terms of it degree however, the optimal of these metrics is matter of real concern as they must be chosen in such a manner that they must measure the true image and state of the software components with respect to the basic principles of software stability with openness for further change [2].

The testing stage plays very important role to ensure the quality of the software products in software development life cycle model, but it is the most resource consuming step in terms of time, effort, and costs. This testing activity represents 50 to 70 percent of the total costs of a project [3]. To reduce these resources one has to maintain our source code and also maintain its metrics.

These resources also depend on complexity of source code. If program is very complex, it will be difficult to understand by the programmer. Complexity plays a very important role to decide the effort, costs, and time of any project or software. Sometimes complex program can be understood easily by the computer or machine but it may be hard to understand by the programmer.

The main goal of this analysis study is to improve the quality of software. Improving the quality of this software implies a reduction in development and maintenance costs, hence a reduction in the costs of the whole company. Higher quality software might also increase customer satisfaction and assurance in the company and its products [4]. To carry out this improvement this study will focus on computing software metrics over the software's source code and relationship among these metrics. This correlation study of metrics will help ones to measures the strength of relationships between two metrics, to regression analysis, which determines the mathematical expression of the relationship [5].

\section{SYSTEM DESCRIPTION}

Data were collected from three projects $\mathrm{P} 0, \mathrm{P} 1$, and $\mathrm{P} 2$ developed by us in JAVA programming language which is object oriented programming language and compiled [6] as well as tested on different tools and other details of these projects are given below.

\subsection{LOC Details of System}

In project P0 authors used 10 classes, 375 Source Lines Of Code (SLOC), 163 lines for comments, 115 blank lines, and this total is 653 .

Table 1. Detail description of system

\begin{tabular}{|c|c|c|c|c|c|}
\hline Projects & SLOC & Comments & $\begin{array}{c}\text { Blank } \\
\text { Lines }\end{array}$ & $\begin{array}{c}\text { Total } \\
\text { LOC }\end{array}$ & Classes \\
\hline P0 & 375 & 163 & 115 & 653 & 10 \\
\hline P1 & 433 & 168 & 118 & 719 & 20 \\
\hline P2 & 494 & 168 & 168 & 830 & 30 \\
\hline Subtotal & 1302 & 499 & 401 & 2202 & 60 \\
\hline
\end{tabular}

In project $\mathrm{P} 1$ authors used 20 classes, 433 Source Lines Of Code (SLOC), 168 lines for comments, 118 blank lines, and this total is 719 . In project P2 authors used 30 classes, 494 Source Lines Of Code (SLOC), 168 lines for comments, 168 blank lines, and this total is 830 . So in this way authors 
analysed 60 classes and 2202 Lines of Code in their whole system. This analysis report is depicted in Table 1.

\subsection{Functional Details of System}

\subsubsection{Project $P O$}

This project has 10 classes. There is no inheritance in any class. Every class has different complexity level. The detail description of these classes is given in Table 2.

Table 2. Detail description of project $P 0$

\begin{tabular}{|c|c|}
\hline Class Name & Description \\
\hline PercentageCalculation1 & This class contains simple variables \\
\hline PercentageCalculation2 & This class contains 1 for loop \\
\hline PercentageCalculation3 & This class contains 1 for loop and if else \\
\hline PercentageCalculation 4 & $\begin{array}{l}\text { This class contains } 1 \text { for loop, if else, } \\
\text { and if else inside for loop }\end{array}$ \\
\hline PercentageCalculation5 & $\begin{array}{l}\text { This class contains } 1 \text { for loop, if else, if } \\
\text { else inside for loop, and } 1 \text { other function } \\
\text { GradeDisplayFunction() }\end{array}$ \\
\hline PercentageCalculation6 & $\begin{array}{l}\text { This class contains } 1 \text { for loop, if else, if } \\
\text { else inside for loop, and } 2 \text { other } \\
\text { functions GradeDisplayFunction(), } \\
\text { PercentageCalculateFunction() }\end{array}$ \\
\hline PercentageCalculation7 & $\begin{array}{l}\text { This class contains } 1 \text { for loop, if else, if } \\
\text { else inside for loop, and } 3 \text { other } \\
\text { functions GradeDisplayFunction(), } \\
\text { PercentageCalculateFunction() and third } \\
\text { DisplayGradeMessage() inside } \\
\text { GradeDisplayFunction() }\end{array}$ \\
\hline PercentageCalculation 8 & $\begin{array}{l}\text { This class contains } 1 \text { for loop, if else, if } \\
\text { else inside for loop, and } 4 \text { other } \\
\text { functions GradeDisplayFunction(), } \\
\text { PercentageCalculateFunction() and third } \\
\text { DisplayGradeMessage() inside } \\
\text { GradeDisplayFunction() } \\
\text { IsTotalMarksConCBOionSatisfied() }\end{array}$ \\
\hline PercentageCalculation9 & $\begin{array}{l}\text { This class contains } 1 \text { for loop, if else, if } \\
\text { else inside for loop, and } 3 \text { other } \\
\text { functions GradeDisplayFunction(), } \\
\text { PercentageCalculateFunction() and third } \\
\text { DisplayGradeMessage() inside } \\
\text { GradeDisplayFunction() and switch case }\end{array}$ \\
\hline PercentageCalculation 10 & This class contains 2 nested for loop \\
\hline
\end{tabular}

\subsubsection{Project PI}

This project has 10 classes. There is single level inheritance. Every class has different complexity level. The detail description of these classes is given in Table 3 .

Table 3. Detail description of project P1

\begin{tabular}{|l|l|}
\hline \multicolumn{1}{|c|}{ Class Name } & \multicolumn{1}{|c|}{ Description } \\
\hline I1PercentageCalculation1 & $\begin{array}{l}\text { This program has single level } \\
\text { inheritance }\end{array}$ \\
\hline I1PercentageCalculation2 & $\begin{array}{l}\text { This program has single level } \\
\text { inheritance and 1 for loop }\end{array}$ \\
\hline I1PercentageCalculation3 & $\begin{array}{l}\text { This program has single level } \\
\text { inheritance and 1 for loop and if } \\
\text { else }\end{array}$ \\
\hline I1PercentageCalculation4 & $\begin{array}{l}\text { This program has single level } \\
\text { inheritance and 1 for loop, if else, }\end{array}$ \\
\hline
\end{tabular}

\begin{tabular}{|c|c|}
\hline & and if else inside for loop \\
\hline I1PercentageCalculation5 & $\begin{array}{l}\text { This program has single level } \\
\text { inheritance and } 1 \text { for loop, if else, } \\
\text { if else inside for loop, and } 1 \text { other } \\
\text { function GradeDisplayFunction() }\end{array}$ \\
\hline I1PercentageCalculation6 & $\begin{array}{l}\text { This program has single level } \\
\text { inheritance and } 1 \text { for loop, if else, } \\
\text { if else inside for loop, and } 2 \text { other } \\
\text { functions GradeDisplayFunction(), } \\
\text { PercentageCalculateFunction() } \\
\text { function in parent class }\end{array}$ \\
\hline I1PercentageCalculation7 & $\begin{array}{l}\text { This program has single level } \\
\text { inheritance and } 1 \text { for loop, if else, } \\
\text { if else inside for loop, and } 3 \text { other } \\
\text { functions GradeDisplayFunction(), } \\
\text { PercentageCalculateFunction() and } \\
\text { 3rd DisplayGradeMessage() inside } \\
\text { GradeDisplayFunction(); } \\
\text { function in parent class }\end{array}$ \\
\hline I1PercentageCalculation8 & $\begin{array}{l}\text { This program has single level } \\
\text { inheritance and } 1 \text { for loop, if else, } \\
\text { if else inside for loop, and } 4 \text { other } \\
\text { functions GradeDisplayFunction(), } \\
\text { PercentageCalculateFunction() and } \\
\text { 3rd DisplayGradeMessage() inside } \\
\text { GradeDisplayFunction(), } \\
\text { IsTotalMarksConCBOionSatisfied; } \\
4 \text { function in parent class }\end{array}$ \\
\hline I1PercentageCalculation9 & $\begin{array}{l}\text { This program has single level } \\
\text { inheritance and } 1 \text { for loop, if else, } \\
\text { if else inside for loop, and } 4 \text { other } \\
\text { functions } \\
\text { GradeDisplayFunction(), } \\
\text { PercentageCalculateFunction() and } \\
\text { 3rd DisplayGradeMessage() inside } \\
\text { GradeDisplayFunction(), } \\
\text { IsTotalMarksConCBOionSatisfied; } \\
4 \text { function in parent class and } \\
\text { switch cases }\end{array}$ \\
\hline I1PercentageCalculation 10 & $\begin{array}{l}\text { This program has single level } \\
\text { inheritance and } 2 \text { nested for loops }\end{array}$ \\
\hline
\end{tabular}

\subsubsection{Project P2}

This project has 10 classes. There are two level inheritances. Every class has different complexity level. The detail description of these classes is given in Table 4.

Table 4. Detail description of project P2

\begin{tabular}{|l|l|}
\hline \multicolumn{1}{|c|}{ Class Name } & \multicolumn{1}{|c|}{ Description } \\
\hline I2PercentageCalculation1 & This program has 2 level inheritance \\
\hline I2PercentageCalculation2 & $\begin{array}{l}\text { This program has 2 level inheritance } \\
\text { and 1 for loop }\end{array}$ \\
\hline I2PercentageCalculation3 & $\begin{array}{l}\text { This program has 2 level inheritance } \\
\text { and 1 for loop and if else }\end{array}$ \\
\hline I2PercentageCalculation4 & $\begin{array}{l}\text { This program has 2 level inheritance } \\
\text { and if else, and if else inside for loop }\end{array}$ \\
\hline I2PercentageCalculation5 & $\begin{array}{l}\text { This program has 2 level inheritance } \\
\text { and 1 for loop, if else, if else inside for } \\
\text { loop, and 1 other function } \\
\text { GradeDisplayFunction() }\end{array}$ \\
\hline
\end{tabular}




\begin{tabular}{|c|c|}
\hline I2PercentageCalculation6 & $\begin{array}{l}\text { This program has } 2 \text { level inheritance } \\
\text { and } 1 \text { for loop, if else, if else inside for } \\
\text { loop, and } 2 \text { other functions } \\
\text { GradeDisplayFunction(), } \\
\text { PercentageCalculateFunction() }\end{array}$ \\
\hline I2PercentageCalculation7 & $\begin{array}{l}\text { This program has } 2 \text { level inheritance } \\
\text { and } 1 \text { for loop, if else, if else inside for } \\
\text { loop, and } 3 \text { other functions } \\
\text { GradeDisplayFunction(), } \\
\text { PercentageCalculateFunction() and 3rd } \\
\text { DisplayGradeMessage() inside } \\
\text { GradeDisplayFunction(); } 3 \text { function in } \\
\text { parent class }\end{array}$ \\
\hline I2PercentageCalculation8 & $\begin{array}{l}\text { This program has } 2 \text { level inheritance } \\
\text { and } 1 \text { for loop, if else, if else inside } \\
\text { for loop, and } 3 \text { other functions } \\
\text { GradeDisplayFunction(), } \\
\text { PercentageCalculateFunction() and 3rd } \\
\text { DisplayGradeMessage() inside } \\
\text { GradeDisplayFunction(); } 3 \text { function in } \\
\text { parent class }\end{array}$ \\
\hline I2PercentageCalculation9 & $\begin{array}{l}\text { This program has } 2 \text { level inheritance } \\
\text { and } 1 \text { for loop, if else, if else inside for } \\
\text { loop, and } 4 \text { other functions } \\
\text { GradeDisplayFunction(), } \\
\text { PercentageCalculateFunction() and 3rd } \\
\text { DisplayGradeMessage() inside } \\
\text { GradeDisplayFunction(), } \\
\text { IsTotalMarksConCBOionSatisfied; } 4 \\
\text { function in parent class and switch } \\
\text { cases }\end{array}$ \\
\hline I2PercentageCalculation 10 & $\begin{array}{l}\text { This program has } 2 \text { level inheritance } \\
\text { and } 2 \text { nested for loops }\end{array}$ \\
\hline
\end{tabular}

\section{EMPERICAL STUDY}

\subsection{Proposed Methodology}

\section{Table 5. Proposed Methodology}

\begin{tabular}{|c|c|}
\hline Steps & Description \\
\hline 1 & $\begin{array}{l}\text { Project } \mathrm{P} 0, \mathrm{P} 1 \text { and } \mathrm{P} 2 \text { are written in java programming } \\
\text { language (Object Oriented Programming Language). }\end{array}$ \\
\hline 2 & Every project is compiled and tested [6]. \\
\hline 3 & $\begin{array}{l}\text { CBO, MVG, and SLOC metrics of project P0, P1, and } \\
\text { P2 are calculated using CCCC, NetBeans } 8.0 \text {, Source } \\
\text { Code Monitor etc. }\end{array}$ \\
\hline 4 & Step $4,5,6,7$ and 8 are done on MATLAB software. \\
\hline 5 & Correlation metrics of project $\mathrm{P} 0, \mathrm{P} 1$ and $\mathrm{P} 2$ are found. \\
\hline 6 & $\begin{array}{l}\text { Mathematical expressions of these metrics are } \\
\text { calculated. }\end{array}$ \\
\hline 7 & Graphs of these metrics are drawn. \\
\hline 8 & $\begin{array}{l}\text { GOF (Goodness OF Fit (SSE and R-Square)) of above } \\
\text { graphs are calculated. }\end{array}$ \\
\hline 9 & The best graph of metrics according to GOF is found. \\
\hline 10 & $\begin{array}{l}\text { Threshold value of metrics is calculated according to } \\
\text { above graphs. }\end{array}$ \\
\hline
\end{tabular}

\subsection{Calculation of CBO Metric}

Authors calculated $\mathrm{CBO}$ of these three projects separately using CCCC software [7]. There are following three tables in this subsection which consist of $\mathrm{CBO}$ values of each project.

\subsubsection{Average $C B O$ analysis of project $P O$}

Table 6. CBO of project $\mathrm{PO}$

\begin{tabular}{|c|c|}
\hline Module Name & CBO \\
\hline PercentageCalculation1 & 1 \\
\hline PercentageCalculation10 & 1 \\
\hline PercentageCalculation2 & 1 \\
\hline PercentageCalculation3 & 1 \\
\hline PercentageCalculation4 & 1 \\
\hline PercentageCalculation5 & 1 \\
\hline PercentageCalculation6 & 1 \\
\hline PercentageCalculation7 & 1 \\
\hline PercentageCalculation8 & 1 \\
\hline PercentageCalculation9 & 1 \\
\hline AVERAGE & 1 \\
\hline
\end{tabular}

\subsubsection{Average $C B O$ analysis of project $P 1$}

Table 7. CBO of project P1

\begin{tabular}{|c|c|}
\hline Module Name & CBO \\
\hline ChildI1PercentageCalculation1 & 2 \\
\hline ChildI1PercentageCalculation 10 & 2 \\
\hline ChildI1PercentageCalculation2 & 2 \\
\hline ChildI1PercentageCalculation3 & 2 \\
\hline ChildI1PercentageCalculation4 & 2 \\
\hline ChildI1PercentageCalculation5 & 2 \\
\hline ChildI1PercentageCalculation6 & 2 \\
\hline ChildI1PercentageCalculation7 & 2 \\
\hline ChildI1PercentageCalculation8 & 2 \\
\hline ChildI1PercentageCalculation9 & 2 \\
\hline I1PercentageCalculation 1 & 1 \\
\hline I1PercentageCalculation 10 & 1 \\
\hline I1PercentageCalculation2 & 1 \\
\hline I1PercentageCalculation3 & 1 \\
\hline I1PercentageCalculation 4 & 1 \\
\hline I1PercentageCalculation5 & 1 \\
\hline I1PercentageCalculation6 & 1 \\
\hline I1PercentageCalculation7 & 2 \\
\hline I1PercentageCalculation8 & 2 \\
\hline I1PercentageCalculation9 & 2 \\
\hline AVERAGE & 1.65 \\
\hline
\end{tabular}




\subsubsection{Average $C B O$ analysis of project $P 2$}

Table 8. CBO of project $P 2$

\begin{tabular}{|c|c|}
\hline Module Name & CBO \\
\hline ChildI2PercentageCalculation1 & 2 \\
\hline ChildI2PercentageCalculation 10 & 2 \\
\hline ChildI2PercentageCalculation2 & 2 \\
\hline ChildI2PercentageCalculation3 & 2 \\
\hline ChildI2PercentageCalculation 4 & 2 \\
\hline ChildI2PercentageCalculation5 & 2 \\
\hline ChildI2PercentageCalculation6 & 2 \\
\hline ChildI2PercentageCalculation7 & 2 \\
\hline ChildI2PercentageCalculation8 & 2 \\
\hline ChildI2PercentageCalculation9 & 2 \\
\hline DchildI2PercentageCalculation 1 & 2 \\
\hline DchildI2PercentageCalculation 10 & 2 \\
\hline DchildI2PercentageCalculation2 & 2 \\
\hline DchildI2PercentageCalculation3 & 2 \\
\hline DchildI2PercentageCalculation4 & 2 \\
\hline DchildI2PercentageCalculation5 & 2 \\
\hline DchildI2PercentageCalculation6 & 2 \\
\hline DchildI2PercentageCalculation7 & 2 \\
\hline DchildI2PercentageCalculation8 & 2 \\
\hline DchildI2PercentageCalculation9 & 2 \\
\hline I2PercentageCalculation 1 & 1 \\
\hline I2PercentageCalculation 10 & 1 \\
\hline I2PercentageCalculation2 & 1 \\
\hline I2PercentageCalculation 3 & 1 \\
\hline I2PercentageCalculation 4 & 1 \\
\hline I2PercentageCalculation5 & 1 \\
\hline I2PercentageCalculation6 & 1 \\
\hline I2PercentageCalculation7 & 2 \\
\hline I2PercentageCalculation 8 & 2 \\
\hline I2PercentageCalculation9 & 2 \\
\hline AVERAGE & 1.76667 \\
\hline
\end{tabular}

\subsection{Calculation of MVG Metric}

Authors calculated MVG [8] of these three projects using NetBeans 8.0 IDE [6] software. Here MVG is the cyclomatic complexity of project $\mathrm{P} 0, \mathrm{P} 1$, and $\mathrm{P} 2$. Table 9 depicts the MVG of each project separately.

Table 9. MVG of project P0, P1, and P2

\begin{tabular}{|c|c|}
\hline Projects & MVG \\
\hline P0 & 75 \\
\hline P1 & 86 \\
\hline P2 & 101 \\
\hline
\end{tabular}

\section{CORRELATION ANALYSIS}

\subsection{Correlation between MVG and CBO}

Table 10. MVG of project P0, P1, and P2

\begin{tabular}{|c|c|c|c|}
\hline Projects & Avg CBO & MVG & $\begin{array}{c}\text { MVG } \\
\text { Increased \% }\end{array}$ \\
\hline P0 & 1 & 75 & \\
\hline P1 & 1.65 & 86 & 14.66 \\
\hline P2 & 1.766667 & 101 & 34.6667 \\
\hline
\end{tabular}

\subsection{Graphs between MVG and CBO}

Authors found the graphs between MVG and CBO using the above given data in Table 10. There may be different graphs like polynomial, exponential, power, and sin etc. These graphs are given below one by one which has been drawn on MATLAB (Matrix Laboratory) [9].

i. Exponential 1 (Number of terms=1)

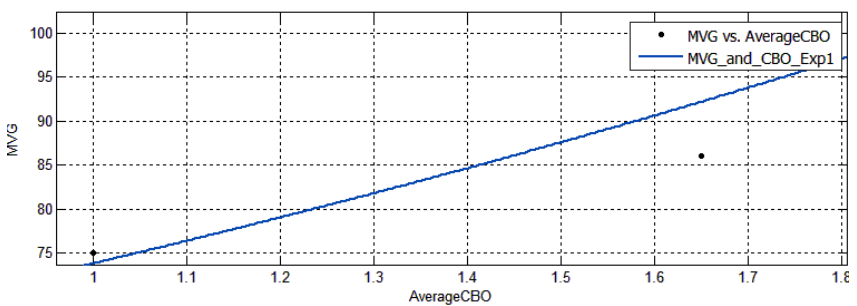

Fig 1: Exponential graph between MVG and CBO

ii. Gaussian 1 (Number of terms=1)

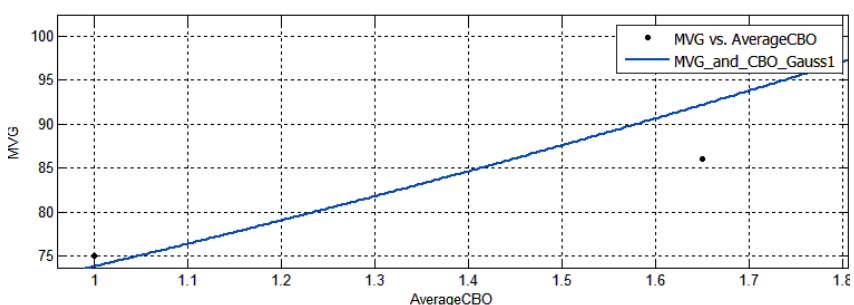

Fig 2: Gaussian 1 graph between MVG and CBO

iii. Polynomial 1 (Degree 1)

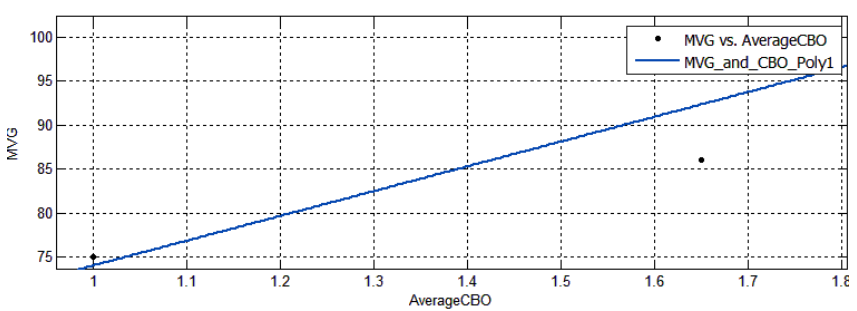

Fig 3: Polynomial 1 graph between MVG and CBO

iv. Polynomial 2 (Degree 2)

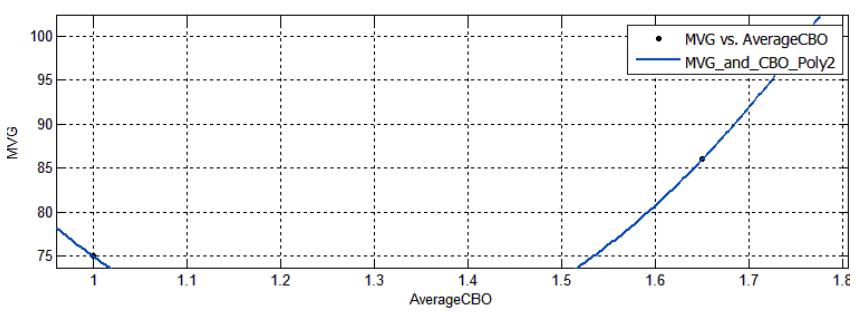


Fig 4: Polynomial 2 graph between MVG and CBO

v. Power 1 (Number of terms=1)

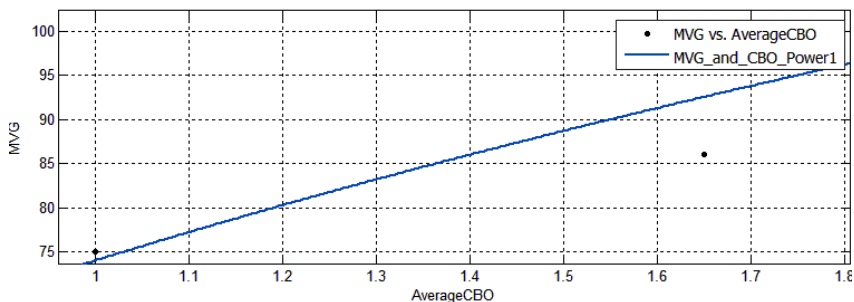

Fig 5: Power 1 graph between MVG and CBO

vi. Power 2 (Number of terms $=2$ )

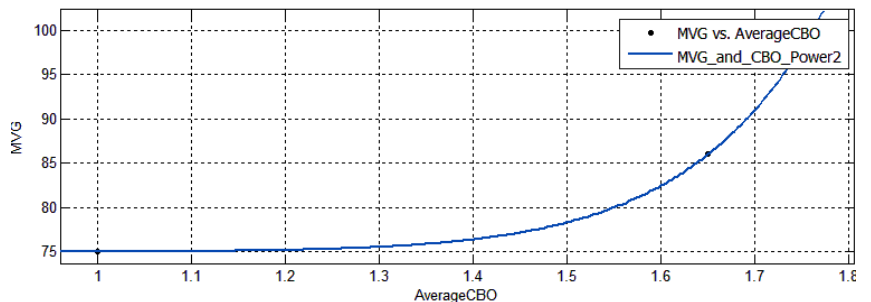

Fig 6: Power 2 graph between MVG and CBO

vii. Rational 01 (Numerator degree $=0$ Denominator degree $=1$ )

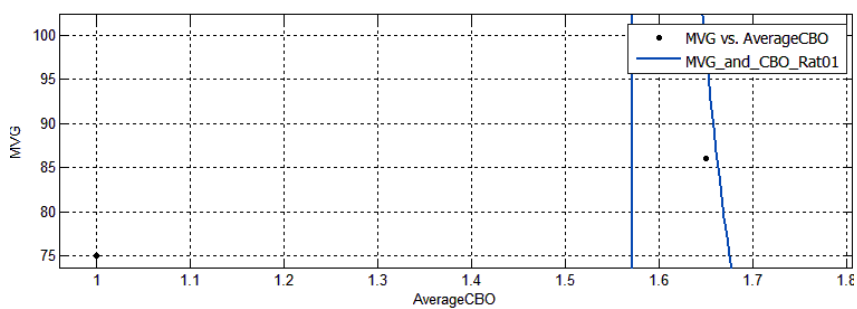

Fig 7: Rational 01 graph between MVG and CBO

viii. Rational 02 (Numerator degree $=0$ Denominator degree $=2$ )

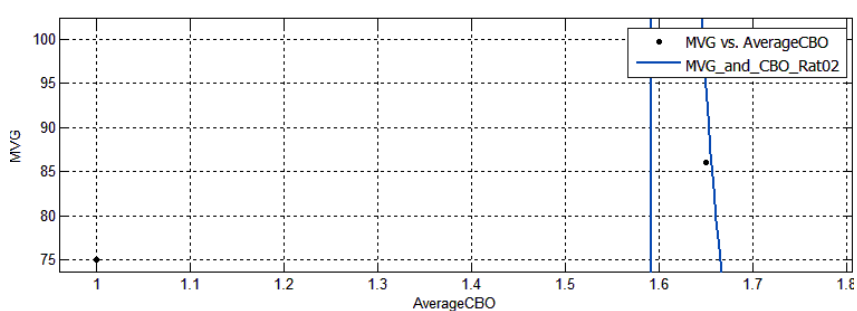

Fig 8: Rational 02 graph between MVG and CBO

ix. Rational 11 (Numerator degree= 1 Denominator degree=1)

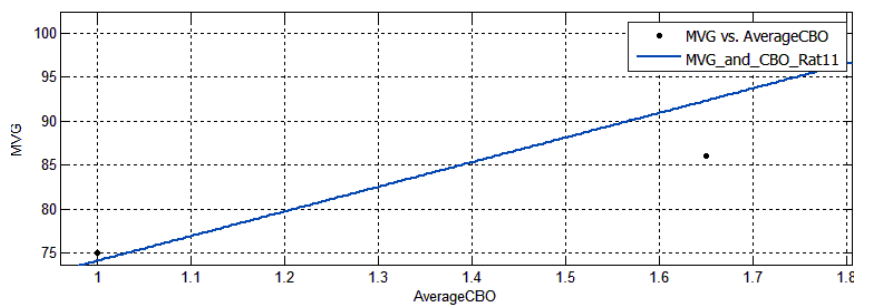

Fig 9: Rational 11 graph between MVG and CBO
X. $\quad$ Sin $1($ Number of terms $=1)$

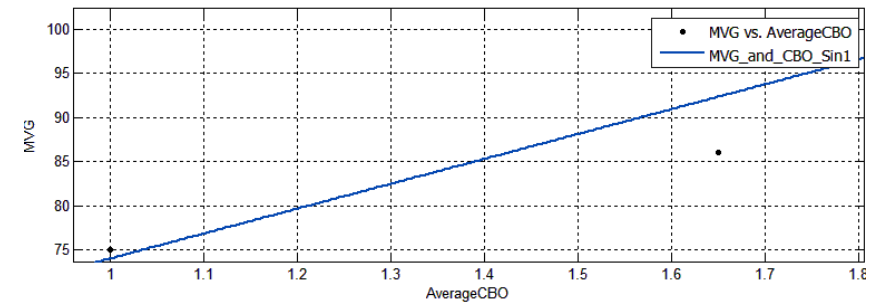

Fig 10: Sin 1 graph between MVG and CBO

xi. Weibull

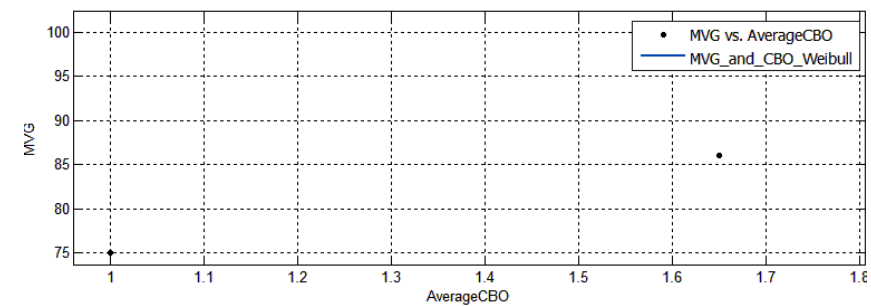

Fig 11: Weibull graph between MVG and CBO

xii. Custom Equation

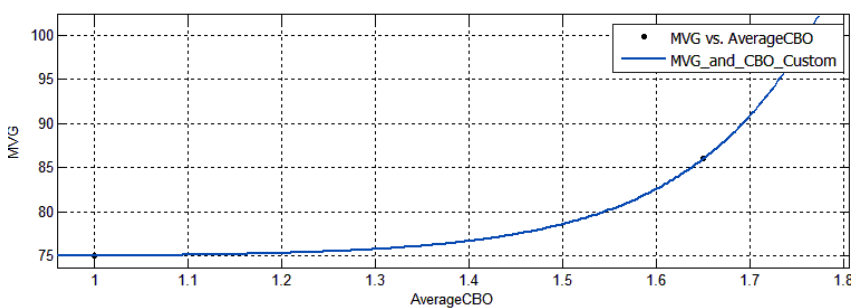

Fig 12: Custom Equation graph MVG and CBO xiii. Mix Graphs

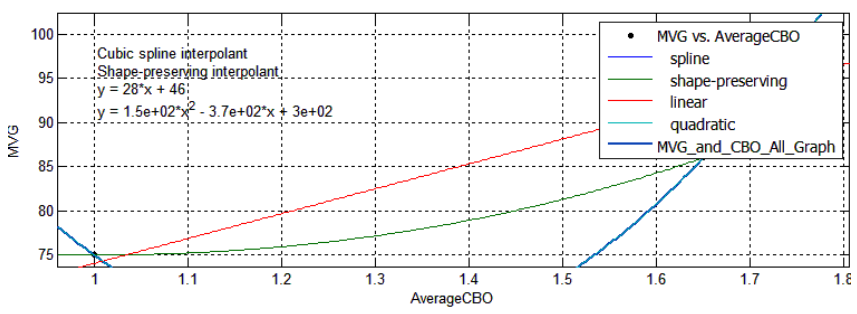

Fig 13: Mix graphs between MVG and CBO

\subsection{Correlation between MVG and CBO}

\subsubsection{Analysis of graphs}

Here authors have calculated mathematical formulas between MVG and CBO. In Table $11 \mathrm{f}(\mathrm{x})$ represents the MVG and $\mathrm{x}$ represents the $\mathrm{CBO}$ of the system [10]. Here Table 11 represents the statistical result of graphs which are sorted in lower error (SSE) to higher error.

If SSE tends to 0 and R-Square tends to 1 then that graph's fit is good. So here Polynomial degree 2 graph is good because it has SSE $=1.94 \mathrm{E}-26$ and $\mathrm{R}$-Square $=1$. 
Table 11. Statistical Result of MVG and CBO metrics

\begin{tabular}{|c|c|c|c|c|}
\hline Fit Type & Formulas & Coefficients & $\begin{array}{l}\text { SSE } \\
\text { (Error) }\end{array}$ & $\begin{array}{l}\text { R- } \\
\text { Square }\end{array}$ \\
\hline Polynomial 2 & $\begin{array}{l}\mathrm{f}(\mathrm{x})=\mathrm{p} 1^{*} \mathrm{x}^{\wedge} 2+ \\
\mathrm{p} 2 * x+\mathrm{p} 3\end{array}$ & $\begin{array}{l}\text { p1 }=145.6 \\
\text { p2 }=-369 \\
\text { p3 }=298.4\end{array}$ & $1.94 \mathrm{E}-26$ & 1 \\
\hline $\begin{array}{l}\text { Custom } \\
\text { Equation }\end{array}$ & $\begin{array}{l}f(x)=a^{*} \exp (- \\
\left.b^{*} x\right)+c\end{array}$ & $\begin{array}{l}a=6.193 e-05 \\
b=-7.331 \\
c=74.91\end{array}$ & $1.71 \mathrm{E}-21$ & 1 \\
\hline Power 2 & $f(x)=a^{*} x^{\wedge} b+c$ & $\begin{array}{l}\mathrm{a}=0.02029 \\
\mathrm{~b}=12.58 \\
\mathrm{c}=74.98\end{array}$ & $2.15 \mathrm{E}-16$ & 1 \\
\hline Exponential 1 & $\begin{array}{l}f(x)= \\
a^{*} \exp \left(b^{*} x\right)\end{array}$ & $\begin{array}{l}\mathrm{a}=52.44 \\
\mathrm{~b}=0.342\end{array}$ & 65.3 & 0.8083 \\
\hline Gaussian 1 & $\begin{array}{l}\mathrm{f}(\mathrm{x})=\mathrm{a} 1 * \exp (- \\
\left.((\mathrm{x}-\mathrm{b} 1) / \mathrm{c} 1)^{\wedge} 2\right)\end{array}$ & $\begin{array}{l}\mathrm{a} 1=3.6 \mathrm{e}+203 \\
\mathrm{~b} 1=2717 \\
\mathrm{c} 1=126\end{array}$ & 65.3 & 0.8083 \\
\hline Polynomial 1 & $f(x)=p 1 * x+p 2$ & $\begin{array}{l}\mathrm{p} 1=28.16 \\
\mathrm{p} 2=45.87\end{array}$ & 70.01 & 0.7945 \\
\hline $\operatorname{Sin} 1$ & $\begin{array}{l}f(x)= \\
\mathrm{a} 1 * \sin (\mathrm{b} 1 * x+c 1)\end{array}$ & $\begin{array}{l}\mathrm{a} 1=1942 \\
\mathrm{~b} 1=0.01452 \\
\mathrm{c} 1=6.307\end{array}$ & 70.02 & 0.7945 \\
\hline Rational 11 & $\begin{array}{l}\mathrm{f}(\mathrm{x})=(\mathrm{p} 1 * \mathrm{x}+ \\
\mathrm{p} 2) /(\mathrm{x}+\mathrm{q} 1)\end{array}$ & $\begin{array}{l}\mathrm{p} 1=2.60 \mathrm{e}+04 \\
\mathrm{p} 2=4.27 \mathrm{e}+04 \\
\mathrm{q} 1=927\end{array}$ & 70.05 & 0.7944 \\
\hline Power 1 & $f(x)=a^{*} x^{\wedge} b$ & $\begin{array}{l}\mathrm{a}=74.02 \\
\mathrm{~b}=0.4462\end{array}$ & 75.06 & 0.7797 \\
\hline Rational 01 & $\begin{array}{l}\mathrm{f}(\mathrm{x})=(\mathrm{p} 1) /(\mathrm{x}+ \\
\mathrm{q} 1)\end{array}$ & $\begin{array}{l}\mathrm{p} 1=8.021 \\
\mathrm{q} 1=-1.568\end{array}$ & $1.1 \mathrm{E}+04$ & -33.51 \\
\hline Rational 02 & $\begin{array}{l}\mathrm{f}(\mathrm{x})=(\mathrm{p} 1) / \\
\left(\mathrm{x}^{\wedge} 2+\mathrm{q} 1^{*} \mathrm{x}+\right. \\
\mathrm{q} 2)\end{array}$ & $\begin{aligned} \mathrm{p} 1 & =2.2 \mathrm{e}+04 \\
\mathrm{q} 1 & =4184 \\
\mathrm{q} 2 & =-6670\end{aligned}$ & $1.2 \mathrm{E}+04$ & -34.37 \\
\hline Weibull & $\begin{array}{l}f(x)=a^{*} b^{*} x^{\wedge}(b- \\
1)^{*} \exp \left(-a^{*} x^{\wedge} b\right)\end{array}$ & $\begin{array}{l}\mathrm{a}=0.03538 \\
\mathrm{~b}=6.305\end{array}$ & $2.3 \mathrm{E}+04$ & -65.63 \\
\hline
\end{tabular}

\section{OBSERVATIONS}

Here authors discussed about the regression analysis of CBO metric with respect to MVG (complexity). It is measured that when one increase CBO, MVG also increases in polynomial form. This paper provides an optimal value of $\mathrm{CBO}$ at which one can have quality software after that value of complexity will increases exponentially.

\section{CONCLUSION AND FUTURE SCOPE}

This regression analysis provides us a monitoring alarm that provides a limit of $\mathrm{CBO}$ at which complexity will increase exponentially. Authors also described graph and mathematical expression between MVG and CBO.
One can do the following work in future.

1. There are many metrics available one can make different combinations and after making the combinations they can find the correlation among those metrics.

2. One can make a system which tells them the relationship among those metrics.

3. One can take our project as reference for various predictions of quality software.

\section{REFERENCES}

[1] S. Dick and A. Kandel. "Fuzzy clustering of software metrics". In Fuzzy Systems, 2003. FUZZ '03. The 12th IEEE International Conference on, volume 1, pages 642 - 647 vol.1, May 2003.

[2] A. Urvashi, and A. Chhabra. "Change-Proneness of Software Components". IOSR Journal of Computer Engineering (IOSR-JCE) Volume 16, Issue 2, Ver. VIII PP 45-48 (Mar-Apr. 2014).

[3] H. Jie Lee, L. Naish, and K. Ramamohanarao. "Study of the relationship of bug consistency with respect to performance of spectra metrics". In 2nd IEEE International Conference on Computer Science and Information Technology, 2009. ICCSIT 2009., pages 501 -508, Aug. 2009.

[4] C. Jin, Shu-Wei Jin, Jun-Min Ye, and Qing-Guo Zhang. "Quality prediction model of object-oriented software system using computational intelligence". In Power Electronics and Intelligent Transportation System (PEITS), 2009 2nd International Conference on, volume 2, pages $120-123$, Dec. 2009

[5] C. Zhang; Budgen, D., "What Do We Know about the Effectiveness of Software Design Patterns?" Software Engineering, IEEE Transactions on, vol.38, no.5, pp.1213, 1231, Sept.-Oct. 2012.

[6] NetBeans IDE 8.0, Available at: "https://netbeans.org/downloads/start.html?platform=win dows\&lang=en\&option=javaee".

[7] $\mathrm{C}$ and C++ Code Counter, Available at: "http://sourceforge.net/projects/cccc/".

[8] T.McCabe, "A complexity measure", IEEE Transactions on Software Engineering, Vol. 2, pp. 308-320, Dec 1976.

[9] MathWorks Products, Available at: "http://www.mathworks.in/downloads/web_downloads".

[10] Harrison, R. Counsell, S. Nithi, R. "Coupling metrics for object-oriented design" Fifth International Conference IEEE 20-21 Nov 1998. 